ÉGYPTE monde arabe

\section{Égypte/Monde arabe}

3| 1990

Médiateur et métaphores 2

\title{
Les logiques associatives dans la communauté arménienne d'Égypte
}

Anne Kazazian

\section{CpenEdition}

\section{Journals}

Édition électronique

URL : https://journals.openedition.org/ema/233

DOI : $10.4000 /$ ema.233

ISSN : 2090-7273

Éditeur

CEDEJ - Centre d'études et de documentation économiques juridiques et sociales

Édition imprimée

Date de publication : 30 septembre 1990

ISSN : 1110-5097

Référence électronique

Anne Kazazian, « Les logiques associatives dans la communauté arménienne d'Égypte », Égypte/ Monde arabe [En ligne], 3 | 1990, mis en ligne le 08 juillet 2008, consulté le 07 juillet 2022. URL : http:// journals.openedition.org/ema/233; DOI : https://doi.org/10.4000/ema.233

Ce document a été généré automatiquement le 7 juillet 2022.

Tous droits réservés 


\title{
Les logiques associatives dans la communauté arménienne d'Égypte
}

\author{
Anne Kazazian
}

1 La communauté arménienne d'Égypte, bien que de taille plus modeste que les communautés grecque, italienne ou juive ${ }^{1}$, joue un rôle de médiation technique, linguistique très important dès l'émergence de l'état moderne égyptien au début du XIX ${ }^{\mathrm{e}}$ siècle, médiation suscitée " par le haut » c'est-à-dire par Muhammad Ali lui-même. Dès le dernier tiers du xix $x^{e}$ siècle cependant, la médiation se déplace, pour deux raisons principalement: d'une part, l'État égyptien ne sollicite plus les communautés en tant que telles ${ }^{2}$, une classe politique et technique égyptienne étant désormais en place, fruit de la politique éducative commencée sous Muhammad Ali; d'autre part, au même moment, la communauté se constitue en «nation » par le biais d'institutions collectives officielles (structures communautaires) ou non (associations) dont le réfèrent se situe hors d'Égypte. Ces institutions, exprimant la volonté de reproduction d'une conscience de soi, créent une série de médiations dans et pour la communauté.

2 Il pourrait sembler paradoxal qu'au moment où la communauté arménienne d'Égypte (ou du moins, quelques-uns de ses membres) occupe une place remarquée au sein de l'État égyptien, sous l'impulsion de Muhammad 'Ali principalement, donc jusqu'aux années 1850 , il n'existe pas à proprement parler de vie associative dans la communauté. Seul un comité pour les pauvres est attaché à l'église selon la tradition, ainsi qu'une école de paroisse (tebradoun), qui n'est pas l'oeuvre de l'église mais d'un particulier, Yeghiazar. Celui-ci fonde l'école en 1828 et c'est encore sous les auspices d'un autre notable. Garabed Agha Kaloust, que l'école - à laquelle il attribue par testament des revenus fixes provenant de terres sera agrandie et transférée en 1854. Pourtant, entre temps (en 1839) l'église de Bayn al-Surayn est construite et s'y ajoute, en 1853, le Patriarcat, appelé ainsi improprement à l'imitation des Patriarcats copte et grec d'Égypte puisque, par rapport à la hiérarchie religieuse arménienne, c'est une prélature (aratchnordaran) qui est établie en Égypte. Le Patriarcat, toutefois, ne semble pas disposer de beaucoup de moyens financiers. 
3 Pour expliquer cette absence de vie associative, il faut revenir avant le début du XIXe siècle. En Égypte, les Arméniens sont principalement artisans ${ }^{3}$, et la communauté y est décrite comme étant de petite taille, d'importance économique modeste. L'immigration encouragée par Muhammad 'Ali est constituée principalement d'artisans qualifiés, d'ingénieurs, de fonctionnaires. La position privilégiée de l'Égypte au sein de ce réseau incite également de grands commerçants à s'y installer: Boghos Youssoufian Bey, originaire de Smyrne, ministre des Affaires Etrangères de Muhammad 'Ali de 1808 à 1844 , fait venir auprès de lui, pour le mettre au service du souverain, son neveu Nubar, qui deviendra Nubar Pacha. Il n'est pas rare de trouver plusieurs membres de la même famille exercer leurs activités sur le pourtour de la Méditerranée, de Trieste, Venise, Marseille à Smyrne ou Alexandrie. L'Égypte, grâce à Muhammad 'Ali, devient une destination choisie non seulement par intérêt économique mais aussi parce que l'égalité y règne, ce que souligne Nubar Pacha dans ses Mémoires : « Le vrai Muhammad 'Ali est celui qui, en Égypte, a renversé toutes ces idées courantes en Orient, toutes ces manières de penser différentes de celles de l'Europe, qui a fait du chrétien l'égal du Musulman et du raya [ "sujet »; ndlr], l'être inférieur, l'égal du bey kurde ${ }^{4}$. »

4 Les Arméniens de ce début du XIX $^{\mathrm{e}}$ siècle $^{5}$ qui ont participé aux différents gouvernements et qui nous ont laissé des traces par leurs écrits ${ }^{6}$ ne révèlent que leur appartenance à cet État moderne en train de se mettre en place et leur souci de contribuer à cette élaboration. Ils ne nous apprennent rien de leur appartenance à la communauté arménienne sinon des bribes, comme le fait par exemple Nubar Pacha dans ses Mémoires (qui ne sont peut-être pas le lieu où pourrait s'exprimer une telle appartenance), quand il :dit: «J'avais un homme dans mon cabinet. Arménien comme moi »... Comme le fait par ailleurs remarquer R. llbert ${ }^{7}$, l'Égypte de Muhammad 'Ali pouvait permettre de penser que « les différents groupes communautaires auraient pu se fondre petit à petit dans une sorte de plus grand commun dénominateur » qui eût été la «nationalité ottomane». Même si les alliances matrimoniales restaient principalement arméniennes, si les, échanges économiques s'opéraient d'abord entre Arméniens, cela n'impliquait pas encore une « conscience de soi » et des moyens mis au service de celle-ci. Détail révélateur, l'élite arménienne (comme l'élite en général) parlait turc ou français tandis que l'ensemble des Arméniens parlaient arabe : c'est ce que révèle une lettre du Patriarcat de la fin du XIx ${ }^{e}$ siècle, demandant "un prêtre sachant parler l'arabe, nos nationaux ne parlant pas l'arménien ». Topouzian ${ }^{8}$, lui, signale pour la même époque la difficulté de l'apprentissage de l'arménien à l'école. Usage de langues qui rend compte d'un clivage de classes, dans la mesure où seules les couches populaires envoyaient leurs enfants à l'école rattachée au Patriarcat, alors que les notables préféraient envoyer les leurs dans les écoles de mission ou à l'étranger.

Comment, dans ces conditions, espérer trouver des associations arméniennes ou une vie associative? En amont de l'immigration en Égypte, dans l'Empire Ottoman central, en Turquie, c'est seulement dans les années 1860 que naît, progressivement, en réaction aux atteintes à la liberté, à la vie même des Arméniens, le réveil de la «nation », terme qui donne un contenu politique au vieux terme de milla (communauté ethnique et/ou confessionnelle). C'est ainsi que la "Constitution de la Nation arménienne " de 1863, qui ne se met pas en place en Égypte sans y susciter de résistances, commence à introduire dans les statuts de la communauté l'idée d'un devoir envers sa nation ${ }^{9}$. Cette Constitution distingue la communauté arménienne des autres communautés en Égypte et l'inscrit dans un réseau dépendant de Constantinople 
(l'Égypte est considérée comme une province par rapport à l'administration centrale arménienne de même que sont considérées comme provinciales les institutions égyptiennes). Il faut dire que contrairement aux Grecs, qui possèdent une véritable nation depuis 1830, les Arméniens ne peuvent développer d'institutions que dans le cadre de la communauté, autour du Patriarcat (cf. IlberT R.: Qui est Grec ?.... Voir note1).

6 La Constitution établit une assemblée au-dessus du Prélat : «L'Autorité représentative déléguée par la Nation s'appelle Administration nationale (...) Il est nécessaire que l'Administration soit représentative pour être nationale.» Les institutions où se retrouvent les « députés » sont le conseil de la Communauté (ou assemblée générale), le conseil civil, le conseil ecclésiastique ainsi que des comités spécialisés (comité des écoles, comité de bienfaisance...) Elle recommande de «veiller à l'amélioration des écoles nationales, d'encourager les associations formées pour I'éducation des enfants des deux sexes "; elle reconnaît comme étant aptes à participer aux structures officielles de la communauté "les censitaires et les capacités", en entendant par « capacités : les fonctionnaires du gouvernement dans les départements ministériels ou autres; les médecins diplômés ; les auteurs d'oeuvres philanthropiques, les instituteurs des écoles et les personnes qui rendent des services à la Nation", ce qui souligne la nécessité de la participation à la création et au maintien de la "Nation». Elle conclut, dans le chapitre «Contributions nationales »: «Tout Arménien qui est majeur et qui exerce quelque profession doit contribuer aux frais de la Nation (...) Le paiement est annuel et sa qualité est fixée d'après les ressources et moyens de chacun. »

7 En établissant officiellement un espace collectif où chacun, ou presque (compte tenu des conditions liées aux années de résidence en Égypte, ce qui n'est pas sans importance dans la mesure où, à la fin du XIX siècle, une grande partie de la population est constituée de réfugiés) peut participer, la Constitution crée une situation qui va rendre possible, y compris hors des structures de la Communauté, le fait de s'associer. C'est sans doute ce qui explique que sa mise en place ait été si conflictuelle. Si le premier conseil civil est établi en Égypte en 1869 et fonctionne jusqu'en 1873, il est suspendu à cette date et ne reprend qu'en 1879 , après révocation de l'archevêque en place.

8 Le deuxième élément qui va rendre possible une vie associative au sein de la communauté arménienne d'Égypte, ce sont les réfugiés. Immigrants d'une toute autre nature que ceux du début du XIX siècle, ils ne sont plus «appelés » en Égypte mais y arrivent chassés de Turquie par des conditions de vie de plus en plus difficiles. L'Égypte est bien souvent un deuxième lieu d'émigration, après celle qui a conduit à Constantinople. Les nouveaux arrivants - du moins ceux qui immigrent juste après 1896 - sont des hommes plus politisés, conscients des conditions qui les ont poussés au départ. De plus, ils ne parlent pas l'arabe. Tout va donc les inciter à se réunir, et pas seulement pour s'entraider. Les idées de la renaissance arménienne avaient déjà trouvé à s'exprimer en Égypte, notamment grâce à un des premiers directeurs de l'école. Apraham Mouradian, fondateur du journal Armaveni, premier journal arménien d'Égypte, en 1865. Les massacres de 1894 et 1896 vont contribuer, de la part de ceux qui arrivent comme réfugiés, à sensibiliser ceux qui ne connaissent pas la situation, puis à les organiser politiquement, car pour les réfugiés un espoir de retour subsiste.

Une vie associative multiforme 
9 Si la situation de la communauté arménienne est en train de changer, tant dans ses institutions que dans sa composition, l'Égypte également change. Une classe politique égyptienne s'est mise en place progressivement et une distinction apparaît entre « Égyptiens » et " originaires de l'empire ottoman », ce que montre R. llbert à travers l'exemple du Majlis al-Chura, créé en 1866 par Isma'il, assemblée dont les membres sont exclusivement Égyptiens. Or, était « Égyptien tout sujet ottoman établi en Égypte avant le $1^{\text {er }}$ janvier 1848 et y ayant conservé un domicile. "C'était le cas de la première immigration arménienne; ce n'est plus celui des réfugiés de la fin du XIX ${ }^{e}$ siècle qui n'arrivent pas de toutes façons pour s'installer mais pensent encore - et pensèrent longtemps, parfois même après le traité de Lausanne de 1923 - rentrer chez eux en Turquie.

10 L'égyptianisation de la fonction publique ainsi que l'impossibilité d'exercer un quelconque monopole économique, selon les termes d'Adalian ${ }^{10}$, comme cela avait pu être le cas sous Muhammad 'Ali, entraînent le repli des notables arméniens sur leur «nation ». Elle en a besoin. A cet égard, la différence entre le destin de Nubar Pacha et celui de son fils, Boghos Nubar, est assez révélatrice. Plusieurs fois ministre, Nubar Pacha avait pu se déclarer passionnément Égyptien ${ }^{11}$ à une époque où être Égyptien était d'une certaine manière être ottoman, les différentes composantes de cette "Nation ottomane » jouissant toutefois d'une égalité de statut, comme il a été dit plus haut; son fils Boghos, lui, sera également un homme politique mais au service de la cause arménienne. C'est lui qui se voit confier en 1912 par le Catholicos - instance religieuse arménienne suprême - la mission d'éclairer les cabinets ministériels et l'opinion publique des pays européens sur les nécessités de réformes des provinces arméniennes; c'est lui également qui dirige la délégation nationale arménienne à la Conférence de la Paix, à Paris, en 1919, en même temps qu'il s'occupe de la communauté arménienne d'Égypte puisqu'il occupe la fonction de Président du Conseil civil.

11 Ainsi se tissent les conditions d'une vie collective dans la communauté ; d'autant que jusqu'à la fin du xix ${ }^{e}$ siècle, il ne semble pas y avoir eu un «quartier arménien » au Caire ou à Alexandrie, ce qui aurait pu créer certaines formes de sociabilité. Si un quartier naît, c'est constitué par les réfugiés, à partir de la fin du XIXe, autour du Patriarcat.

12 Dès ses débuts, vers la fin du XIX ${ }^{e}$ siècle, la vie associative exprime une variété qui reflète les préoccupations et les activités d'une population hétérogène. De la «société arménienne des amis de la lecture » (1896) à la " société philharmonique arménienne » (1907) en passant par « la mission des réfugiés arméniens » (1896) et les associations de compatriotes (à partir des années 1920) jusqu'aux Unions de métiers, c'est l'abondance qui frappe.

13 Ces associations sont le fait tant de la "société civile » que de la Communauté - c'est-àdire de sa "représentation nationale» - parce que, comme nous l'avons vu, il entre désormais dans ses prérogatives de s'occuper du bien-être de l'ensemble de ses membres, ce qui signifie, à la fin du XIX ${ }^{\mathrm{e}}$ siècle, héberger, porter assistance et scolariser.

14 Dans le chapitre «Inspectorat des recettes et des dépenses » de la Constitution de la Nation arménienne, texte fondateur des institutions officielles de la communauté arménienne d'Égypte, on trouve inscrits comme revenus éventuels «les contributions nationales, les recettes du greffe du Patriarcat, les dons ou legs faits simplement au 
nom de la Nation » tandis que les dépenses peuvent concerner « les frais ordinaires du Patriarcat, les secours pécuniaires destinés aux nécessiteux des paroisses et aux établissements confiés à l'Administration centrale ». Or, dès le début de la "mise en service » des structures communautaires - différents conseils et comités - les moyens financiers manquent. Une lettre de 1897 fait état d'une trentaine de familles aisées et souligne la précarité des conditions de vie du reste de la communauté. Il est question (affaire soulevée par le Conseil civil) de supprimer l'impôt national et de diminuer les tarifs du greffe du Patriarcat "afin de tout faire pour favoriser la prospérité, les mariages ", plutôt que de les compter comme revenus pour les institutions officielles. Si celles-ci fonctionnent cependant, cela résulte davantage de «bricolages» (accords avec l'hôpital européen pour accepter à prix réduits les Arméniens réfugiés après 1896 : la communauté arménienne n'était ni si grande ni si riche qu'elle pût s'offrir, comme les Grecs ou les Italiens, un hôpital) et appel aux notables; le rôle de bienfaisance est presque entièrement assuré, dès sa création en 1906, par l'Union Générale de bienfaisance, même si existait un comité de bienfaisance au sein des institutions communautaires. Ainsi, une lettre du 20 mars 1904 intitulée «Appel au peuple arménien d'Égypte », requête émanant du Patriarcat et qui a pour but de recueillir de l'argent " afin d'acheter un terrain pour construire une nouvelle école », ce qui sera fait en 1907, précise que "l'école nationale tombe en ruines, ne suffit pas aux enfants des deux sexes de la colonie, qui s'accroît de jour en jour ». Une autre lettre de 1929 énonce les difficultés de l'école «face aux dépenses, à cause du grand nombre d'élèves des classes ouvrières qui n'ont pas les moyens de payer les frais d'études ${ }^{12}$ ".

Certains notables commencent à léguer « au nom de la Nation »; c'est le cas de Krikor Yeghiayan qui, par son testament rédigé en 1906, déclare léguer « une propriété au profit de l'école paroissiale arménienne orthodoxe du Caire (...) qui devra en employer les revenus pour l'institution d'une bourse en faveur des étudiants arméniens pauvres sans distinction de rites - (...) qui auront obtenu leur baccalauréat, afin de leur permettre de faire des études supérieures." Ainsi, ce rôle des institutions communautaires, soutenues par les notables, de veiller à l'éducation de tous, notamment en assurant la gratuité scolaire, contribue à une scolarisation presque complète des enfants de la communauté. C'est grâce aux réfugiés que les écoles du Patriarcat sont fréquentées puisque ce qu'ils recherchent en Égypte c'est d'abord leur "nation", ce qui passe par l'école surtout dès lors qu'elle peut être gratuite. Les demandes d'emploi adressées aux institutions communautaires vers les années 1930-40 pour solliciter des recommandations concernent presque toutes des emplois de bureau, ce qui souligne la réussite de cette politique scolaire.

Même si les institutions officielles de la communauté prennent en charge certains domaines (l'éducation principalement) ce qui est une façon de participer à la vie de la communauté et de l'infléchir, c'est l'apport des immigrants de la fin du XIx ${ }^{\mathrm{e}}$ siècle qui contribue de manière décisive à créer une véritable vie collective, notamment par le biais des associations. Les premières associations culturelles, les premiers clubs (à la fois culturels et politiques) apparaissent d'abord à Alexandrie, sans doute parce que c'est le port où arrivent les immigrants, peut-être aussi pour des raisons qui tiennent à la ville elle-même : c'est toujours de là que viendront les discussions et même les troubles. En 1902 y est fondé le club Tigrane Yergat, lié au Parti Hintchak réformé ${ }^{13}$ : en 1908, c'est le club Hamaskaïne, lié au parti Tachnag c'est-à-dire la Fédération révolutionnaire arménienne, club créé semble-t-il par des révolutionnaires venus du Caucase. Au Caire, la formation de clubs est plus tardive, elle démarre vers les années 
20: club artistique lié au parti Ramgavar, parti libéral-démocrate issu du schisme à l'intérieur du parti Hintchak réformé, club Navassart lié au parti Tachnag. Ces clubs vont structurer la vie de la communauté au point de la fractionner, voire de la déchirer. Il n'y est fait état que des questions arméniennes, ce qui a d'autant plus de sens que la majorité des réfugiés espèrent retourner en Turquie, qu'ils voient leurs espoirs anéantis et qu'ils suivent de près la création de la jeune république arménienne. La chute de celle-ci en 1920 et le ressentiment que l'événement provoque chez les Tachnag, qui se sont fait évincer du pouvoir, par les Bolcheviks, conduit à diverses attitudes dont le refus de reconnaissance, par le parti Tachnag, de la République arménienne soviétique, ce qui n'est pas sans provoquer de nombreuses polémiques et même des bagarres dans la communauté. Ainsi, c'est par rapport à des questions arméniennes que l'on se détermine et que les clivages s'opèrent. Lors du massacre d'Adana, « les chefs spirituels des trois communautés religieuses arméniennes d'Égypte [apostolique, catholique et protestante] avaient convoqué au Caire un meeting pour protester contre ce massacre ». Cela conduit Vahan Malézian, avocat arménien membre du barreau du Caire, à écrire à ce sujet dans un journal égyptien, Le Progrès, pour rectifier "un chef -d'œuvre de mensonge " paru dans Le Journal au Caire, à savoir une lettre du mufti d'Adana en réponse à une fatwa (consultation juridique délivrée par une personnalité religieuse musulmane, ndlr) du Recteur de l'université d'AI-Azhar condamnant les massacres de chrétiens dans la province d'Adana. C'est la même attitude qui incite Monseigneur Mouchegh, archevêque d'Adana en voyage en Égypte au moment des massacres, à publier dans un journal d'Alexandrie, Les Nouvelles, une réponse adressée à l'émir Arslan, qui avait réagi de la même façon que le mufti d'Adana à la condamnation des massacres par le recteur.

17 Si la question arménienne s'exprime hors des journaux arméniens, c'est d'abord pour se faire correctement comprendre. Ainsi le prélat des Arméniens se trouve-t-il obligé d'en référer, en 1926, à Abd al-Khaliq Sarwat Pacha, Président du Conseil des Ministres et Ministre de l'Intérieur égyptien, lors des troubles et des bagarres qui éclatent dans la communauté d'Alexandrie : « II est de mon devoir de porter à votre connaissance que la question qu'au Parlement l'honorable Dr. Mahgoub Bey, député du district de Minet alBassal à Alexandrie, vous a posée relativement au conflit qui trouble ces derniers jours la vie jusqu'à présent si paisible de la communauté arménienne d'Égypte, est fondée sur de fausses informations qui lui ont été fournies. Je prends donc la respectueuse liberté de fournir à votre Excellence quelques éclaircissements à ce sujet». Ces explications traduisent en fait « le mécontentement général de la communauté contre un groupe ne représentant qu'une insignifiante minorité qui a, depuis quelque temps, mis dans un étal déplorable l'Evêché, l'Eglise, l'Ecole et les propriétés de notre communauté d'Alexandrie. Ce sont les partisans de ce groupe (...) qui eurent dernièrement, pendant une nuit, dans la salle des séances de l'Evêché, une bagarre tellement scandaleuse que la police dut intervenir.»

18 Entre 1909 et 1926, le ton change. En 1909, lors de l'explication au sujet des massacres arméniens d'Adana, il pouvait être ferme: «Si le mufti d'Adana n'a pas subi un châtiment mérité avec ses monstrueux collègues, c'est parce qu'à Adana l'échafaud n'a été dressé que pour les victimes (...). La parole, ces derniers jours, est aux bourreaux qui, plus que jamais, restent maîtres de la situation (...) quel paradoxe ! La Réaction et la Constitution se donnant la main par-dessus les cadavres de 30000 hommes. » En 1926, la situation n'est plus la même: les rescapés du génocide de 1915, les réactions arméniennes face à la révolution égyptienne de 1919 (nous en parlerons plus loin) et le 
refus qui s'ensuit de ne pas se mêler de politique égyptienne, le Traité de Lausanne de 1923 qui refuse tout territoire aux Arméniens en Turquie, la dissolution de la République arménienne indépendante transforment radicalement la situation des Arméniens (jusqu'alors en instance de retour), ce qui explique notamment les discussions ou les scissions politiques à l'intérieur des partis arméniens, ainsi que le désir des autorités arméniennes de se rendre « invisibles » face à l'État égyptien tout en manifestant pourtant leur besoin d'une intervention étatique: « Nous serions obligés au gouvernement de bien vouloir nous accorder son précieux concours », écrit le prélat dans une lettre adressée à Abd al-Khaliq Sarwat Pacha en 1926, qui nous apprend que la Communauté arménienne d'Égypte, n'étant plus rattachée au Patriarcat de Constantinople depuis la déclaration de l'indépendance de l'Égypte, aurait dû traiter directement avec le gouvernement égyptien; or celui-ci répond que «le règlement organique de la Communauté arménienne d'Égypte n'étant pas encore reconnu par le gouvernement ", il ne veut pas intervenir dans les affaires privées de la communauté.

De même que les clubs s'occupent des problèmes arméniens, de même les journaux arméniens se réfèrent-ils presque exclusivement à ce qui se passe en Turquie, puis en Arménie indépendante et soviétique. Entre 1865 et 1896 existaient deux journaux. Il s'en crée 78 entre 1896 et 1930, et 45 entre 1930 et 1948 (liste établie par le Dr Souren Bayramian). Même si certains journaux se limitent à un numéro ou ne durent que quelques mois, leur profusion étonne, d'autant que là encore, il n'y est question que d'Arménie, "patrie mentale ${ }^{14}$ " à défaut de patrie véritable. Si, d'un point de vue géographique, l'Arménie soviétique n'est pour presque personne en Égypte la patrie d'origine ( $90 \%$ des réfugiés sont originaires de Turquie et nombreux seront ceux qui, pendant longtemps et même après 1923, n'achèteront aucun bien en Égypte et ne "s'implanteront » pas, pensant qu'ils ont une terre, une maison à Mouch, à Césarée, à Diarkébir), c'est comme " patrie » qu'elle sera défendue par les comités de propagande pour le rapatriement dans les années 1946-47; ce qui, peut expliquer le départ notamment mais pas uniquement - d'une population âgée qui espérait finir ses jours précisément dans une "patrie» (dont on savait définitivement, depuis le Traité de Lausanne, que ce ne pouvait être la Turquie).

20 Si les clubs politiques naissent de l'arrivée de réfugiés après 1896, les associations de compatriotes, elles, apparaissent dans les années 20 comme volonté de se retrouver, de se souvenir, comme expression d'une logique : ne pas disparaître à tout jamais. Ainsi trouve-t-on I' « Union patriotique d'Arabkir », l'« Union des compatriotes arméniens de Césarée et de ses environs ", I'«Union' des compatriotes d'Eguine »... Les unions n'expriment pas, ce que manifestait la création de clubs politiques, un désir de comprendre, de lutter pour revenir au pays, mais le désarroi d'une population qui, rescapée d'une catastrophe, cherche à se retrouver par villes, par villages, sans penser à s'organiser plus avant, sinon par le fait même de se rassembler, de témoigner de ce qui fut.

21 Les associations de bienfaisance pouvaient ainsi être le fait d'unions de compatriotes comme de clubs. Dès la fondation en 1906, par Boghos Nubar, de l'Union Générale Arménienne de Bienfaisance (UGAB) qui, il est vrai, dépasse le cadre égyptien, celle-ci regroupe une grande partie des charges supportées par de plus petites structures. C'est le domaine de la bienfaisance qui révèle un aspect de la vie communautaire qui n'est pas seulement tourné vers la communauté d'Égypte mais où celle-ci se trouve le plus 
inclue dans un réseau. C'est le domaine qui nous fait saisir le mieux le caractère de « diaspora » de la communauté.

De la «souscription pour un village incendié » en Turquie en 1896, à celle, pour le couvent de Fournouz à Zeytoun, "détruit par les événements universellement connus ", et où l'on projette de fonder un orphelinat national en 1900, en passant par l'aide à Adana (1909) ${ }^{15}$, l'aide à l'Arménie "pour sauver Etchmiadzine de la famine » (lieu de résidence du catholicossat arménien apostolique), l'aide "pour la reconstruction de l'Arménie en 1925 ", l'aide aux "sinistrés du tremblement de terre de Chiraz (Arménie) en 1927 », on perçoit, en même temps que l'activité incessante de la communauté - tant du côté des instances officielles que de ses membres - le glissement qui s'opère de la Turquie à l'Arménie comme si, puisqu'il n'y a plus d'Arménie en Turquie (ainsi en a décidé le Traité de Lausanne), il fallait préserver le peu qui reste de centralité (d'autant que ce n'est pas peu aux yeux des Arméniens, puisque c'est en Arménie soviétique que se trouve le catholicossat). En 1924, Boghos Nubar fonde I'« «uuvre des boursiers arméniens », qui se propose d'établir des boursiers en Arménie. La même année, un « annuaire des Arméniens d'Égypte ${ }^{16}$ " propose, après une première page d'allégeance au roi Fouad, puis aux autorités anglaises d'occupation et une deuxième page consacrée à la population de l'Égypte, une troisième page consacrée à la population de l'Arménie, à celle de Géorgie et d'Azerbaïdjan. Raccourcis saisissants de la nouvelle donne politique.

L'ensemble des autres associations («Union des bijoutiers arméniens pour l'éducation des élèves nécessiteux »; «Union des instituteurs arméniens du Caire ", "Les Amis de la lecture », la "société philharmonique » ainsi que les associations sportives) rendent compte, pour terminer, d'une vie sociale à l'intérieur de la communauté, qui peut englober toutes les facettes d'une collectivité sans qu'elles représentent pour autant une spécificité de la communauté arménienne, si ce n'est qu'elles révèlent l'existence de couches sociales "moyennes » (il n'y a pas de Chambre de commerce comme chez les Grecs d'Égypte, ou d'Union d'industriels...), même s'il existe quelques notables très aisés.

Les Arméniens face au nationalisme égyptien

On a souvent dit que les Arméniens avaient participé au mouvement syndicaliste ainsi qu'au parti communiste d'Égypte, deux structures qui étaient, au début du siècle, minoritaires. C'est pourtant le fait d'un petit nombre d'entre eux, ce qui s'explique par les conditions de leur arrivée à la fin du XIX $x^{e}$ siècle.

Le Parti socialiste égyptien, fondé en 1920, est surtout formé de membres de diverses communautés à Alexandrie (Italiens, Grecs, Arméniens, Juifs); au Caire, il est plutôt composé d'Égyptiens. Il devient le Parti communiste égyptien en 1923. Le mouvement syndicaliste lui-même en est à ses débuts. Pour en faire partie, il faut travailler dans le textile, les transports ou les usines de tabac. Au début du siècle, la majorité des Arméniens se répartit entre divers types d'emploi: professions libérales (médecins; avocats) pour une partie de la communauté implantée probablement depuis longtemps, services gouvernementaux ou privés, artisanat - des ateliers de petite taille le plus souvent, mais pas toujours (qui vont de l'atelier d'orfèvre à celui de cordonnier) - ou encore métiers qui ne nécessitent que peu de matériel et pas de local tels que cireurs de chaussures, photographes ambulants... Pour une partie de ceux qui arrivent et ne trouvent pas de travail chez des Arméniens, dont ils parlent la langue, l'usine. 
Dans tous les cas, on peut se demander s'ils étaient en mesure de participer à des mouvements égyptiens (partis politiques ou syndicats), d'une part parce qu'ils ne parlaient pas l'arabe - ce pouvait être le cas également des Grecs ou des Italiens, surtout à Alexandrie (le fait qu'une bonne partie de ses membres ne parlaient pas l'arabe était justement le défaut du premier embryon de Parti communiste égyptien, mais il était constitué d'une élite intellectuelle et sociale, ce qui n'était pas forcément le cas des Arméniens qui arrivaient, surtout après 1915); d'autre part parce que, pour ceux qui étaient en Égypte depuis longtemps comme pour les autres, ils restaient des "étrangers", même locaux, ce qui constituait en fait un paradoxe car ils ne possédaient pas les avantages des étrangers ${ }^{17}$ : ces derniers disposaient d'un consulat et pouvaient dans les cas extrêmes, lors de grèves par exemple, être expulsés d'Égypte alors que les sujets locaux risquaient le même sort que les Égyptiens, tout en n'étant pas considérés de la même façon, notamment dans un contexte nationaliste.

C'est dans ce climat nationaliste qu'éclatent les troubles de 1919, troubles qui sont rapportés comme suit par l'Association égyptienne de Paris :

Nous devons dire un mot du prétendu massacre des Arméniens dont certains journaux ont parlé avec complaisance dans un but facile à deviner [la volonté d'indépendance de l'Égypte]. Dès le début de la guerre, le peuple égyptien fut complètement désarmé ; les proclamations punissaient des peines les plus sévères ceux qui cachaient ou portaient des armes. La population avait obéi aux ordres donnés. Lors des manifestations, les Arméniens seuls avaient des armes, ce qui était étrange et inexplicable. Le peuple égyptien n'a jamais eu d'animosité contre les Arméniens qui jouissent dans notre pays de notre proverbiale hospitalité au même titre que les autres étrangers. On devine donc aisément la stupéfaction générale et l'exaspération de la foule des manifestants lorsque des Arméniens usaient de leurs armes contre eux, fait inexplicable qui coïncidait souvent avec l'arrivée des mitrailleuses anglaises chargées d'étouffer le mouvement à tout prix. Les meurtres commis mettaient les manifestants dans le cas de légitime défense; il se peut que la foule exaspérée ait confondu quelques innocents avec les coupables ${ }^{18}$.

En 1919, il y avait un camp de réfugiés à Héliopolis. Est-ce la panique de certains, l'inconscience politique (ou la trop grande conscience ?) d'autres, qui les conduisit à méconnaître le caractère pacifique de la révolution égyptienne de 1919, essentiellement anti-britannique? Se pouvait-il toutefois, à cette date, que les comportements fussent sereins? En tout cas, l'attitude arménienne, qui reste à éclaircir, montre qu'il ne s'agissait pas, en tout état de cause, d'une véritable prise de position vis-à-vis de la vie politique égyptienne, mais plutôt d'une réaction face à quelque chose que l'on ne comprenait pas ou ne pouvait pas comprendre. Après cette date, on n'entend plus parler des Arméniens en politique. Pourtant, ce n'est pas cette date symbolique qui marque un retrait des Arméniens de la politique égyptienne. Celuici, comme nous l'avons dit, avait commencé dès la seconde moitié du XIXe siècle.

29 Malgré le nationalisme égyptien croissant, les structures égyptiennes restent « ottomanes » jusque vers les années 50, alors même que l'Empire ottoman n'existe plus comme entité politique. Cela permet aux Arméniens, par exemple, d'être en Égypte en tant que tels, c'est-à-dire "originaires de l'Empire ottoman », ce qui leur confère un certain statut : celui .d'étrangers locaux. Les logiques associatives de la communauté se déterminent dans ce flou bienveillant qui reconnaît aux communautés le droit de s'auto-administrer. Les tribunaux de statut personnel ne sont supprimés en Égypte qu'en 1955 et ce n'est ce que dans les années 1955-60 que l'on commence à sentir menacée l'autonomie qui avait été jusque-là préservée. C'est à ce moment, pourtant, 
que la communauté est peut-être installée le plus solidement en Égypte, notamment parce qu'il n'est plus possible d'aller ni en Turquie ni en Arménie soviétique (la déception de ceux qui sont partis - relativement peu nombreux d'Égypte, comparativement à ceux de Syrie, du Liban - est à la mesure de l'espoir qui les avait déterminés à le faire). La plus grande partie des associations, ces mêmes années, sont mises sous tutelle égyptienne (ministère des Affaires sociales pour les associations de bienfaisance, dont les biens sont mis sous séquestre ; ministère de l'Education pour les écoles arméniennes), ce qui pouvait donner à penser qu'elles n'auraient plus la liberté de s'exprimer.

Avec le recul, s'il y eut un très grand nombre de départs d'Égypte, dans les années 60 , les associations, même si le cadre en est égyptien, semblent être restées inchangées. Elles ont retrouvé leur liberté d'action, même si celle-ci ne touche plus grand monde. Les mêmes notables s'occupent de plusieurs associations à la fois (culturelles, sportives, de bienfaisance) comme pour montrer que la collectivité fonctionne encore parfaitement. Les logiques de préservation et de renouvellement de la culture, qui avaient contribué à la naissance de la vie associative, continuent en fait à s'exercer jusqu'à aujourd'hui même si, faute de participants, elles ne reflètent plus la diversité qui avait été la leur. Elles s'organisent autour de deux pôles: d'une part l'Arménie, " centralité » culturelle plus que politique (puisqu'elle est soviétique), fragment de ce qui était le lieu d'origine; d'autre part les États-Unis qui, parce qu'ils disposent de moyens financiers (mais pas seulement) rendent possible la prise en charge d'institutions officielles de la communauté et d'associations, cellules ultimes de l'effectivité de la conscience de soi.

\section{NOTES}

1. La communauté grecque est évaluée en 1840 à 5000 personnes; la communauté italienne à 6000 , la communauté juive entre 5000 et 7000. En 1907, les Grecs sont environ 60 000, les italiens 35 000, les juifs 40000 et les Arméniens 8000 (chiffres donnés par Kramer (G.), The Jews in modern Egypt, 1914-1952, I.B. Tauris, London, 1989. Voir aussi Kitroeff (A.), The Greeks in Egypt. 1919-1937: Ethnicity and class, Ithaca Press, Oxford, 1989. llbert (R), « Qui est Grec ? La nationalité comme enjeu en Égypte (1830-1930) », Relations Internationales, n54, été 1988, pp. 39-160.

2. On pourrait peut-être les retrouver ailleurs, dans la participation à la naissance de la municipalité notamment à Alexandrie, dans un tout autre contexte donc. Cf. Ilbert (R.), Alexandrie, espace et société. 1830-1930 (4 vol.), Thèse E.H.E.S.S., Paris, 1990.

3. Voir à ce sujet Raymond (André), Artisans et commerçants au Caire au XVIIIe siècle, (2 vol.), IFEA, Damas, 1974.

4. Nubar pacha, Mémoires, Introduction et notes de M. Boutros-Ghali, Librairie du Liban, Beyrouth, 1983.

5. «" "Durant la $1^{\text {ère }}$ moitié du siècle, le Divan des Affaires étrangères est surnommé le "Palais Arménien ». Il est dirigé souvent par un ministre arménien ; de surcroît, tous les traducteurs, tous les directeurs et sous-directeurs sont aussi Arméniens. » Cf. 
Boudjikanian (A), « Les rôles socio-économiques et politiques des Arméniens d'Égypte au XIX siècle ", in Economie et sociétés dans l'Empire ottoman (Fin XVIII ${ }^{e}$ - début XXe), Actes du colloque de Strasbourg, 15 juillet 1980, Ed. CNRS, Paris, 1983.

6. II suffit de voir les ouvrages, tous consacrés à l'Égypte, de Yacoub Artin Pacha (L'instruction publique en Égypte, La propriété foncière en Égypte) à Nubar Pacha. Ce sont des ouvrages d'hommes d'État. Par ailleurs, un Annuaire des Arméniens d'Égypte, de Adjemian (L.) (Imprimerie Kassabian, Alexandrie, 1924, en arménien) donne la liste des ministres des Affaires Etrangères d'Égypte depuis Muhammad 'Ali : Boghos Youssoufian Bey (1808-1844 ); Artin Tcherakian Bey (1844-1850) ; Stépan Demirdjian Bey (1850-1853) ; Stepan Demirdjian Bey (1855-1857) ; Nubar Pacha (1866-1874) ; Nubar Pacha (1875-1876) ; Nubar Pacha (1878-1879) ; Nubar Pacha (1884-1888) ; Dikran Abro Pacha (1891-1894).

7. Ilbert (Robert), Alexandrie, espace et société. 1830-1930, (4 vol.), Thèse E.H.E.S.S, Paris, 1990.

8. Topouzian (O. X.), Histoire de la colonie arménienne d'Égypte (1805-1952), Académie des sciences de la RSS d'Arménie, Erevan, 1978 (en arménien).

9. La Constitution avait été accordée en 1863 par le gouvernement ottoman au « milla " introduisait une participation « démocratique » (notabilitaire plutôt, car censitaire) de l'ensemble des Arméniens, y compris ceux des Provinces (c'est en fait pour eux qu'elle avait été réclamée) aux structures communautaires, alors aux mains d'une minorité (Patriarcat et grands commerçants ou financiers essentiellement).

10. Adalian (Rouben), "The Armenian Colony of Egypt during the Reign of Muhammad Ali (1805-1848)", Armenian Review, vol. 33. june 1980, p. 117-139. “Garabed (Kaloustian) was also granted the management of the public baths, a state-owned monopoly. In return for his services, he retained the profits from one days income at the baths per week. (...) In 1816, another Armenian had received for 500 purses the monopoty for the sale of spices. As the result of this monopoly, the anise, the cumin and other items from Upper Egypt were sold at the prices fixed by this man."

11. II aurait souhaité cependant, vers la fin de sa vie, s'occuper des affaires arméniennes : « Le destin m'a conduit tout jeune en Égypte ; j'y ai trouvé des protecteurs aussi puissants que généreux ; la tâche qu'ils m'ont assignée, je l'ai remplie avec enthousiasme, avec conviction et je me suis attaché de coeur à ce pays ; cependant, je n'ai pas oublié que j'étais Arménien. » Si son fils Boghos sembla être " plus Arménien », c'est d'abord parce qu'il eût l'occasion de le montrer. » Cf. Archarouni, Nubar Pacha (s. d. s. e.)

12. Ces deux lettres ont été choisies en fonction de leurs dates : la première après l'arrivée des réfugiés de 1894-96, la seconde après le génocide de 1915 (qui se prolonge finalement jusque dans les années 1925-30 avec les rescapés de Smyrne et les orphelins du Near East Relief).

13. Pastermadjian (H.), Histoire de l'Arménie, Paris, Samuelian, 1986, p.373 : « Le premier comité révolutionnaire arménien fut fondé à Genève en 1887. (...) Il donna naissance au parti Hintchak. (...) Ce fut ce parti qui commença l'épopée révolutionnaire arménienne, sous le nom de Fédération révolutionnaire arménienne ou Daschnatzoutioun, (...) L'activité de ces deux partis révolutionnaires arméniens était à l'origine principalement dirigée vers la défense de la nationalité arménienne dans l'Empire Ottoman. Leurs conceptions étaient basées sur les idées de la démocratie occidentale. Leurs revendications étaient à l'origine des plus modérées. (...) Jusqu'en 1895, le 
nombre des adhérents de ces partis fut limité. Ce furent les massacres qui donnèrent une impulsion décisive au mouvement révolutionnaire arménien ».

14. «Ceux du Caire ont compris qu'il leur fallait s'unir autour d'un patrimoine, d'une idée de patrie à défaut de la patrie même. Et ce patrimoine ne pouvait être qu'intellectuel puisqu'ils avaient dû tout abandonner. Il fallait organiser une défense de la culture, héritage que nul ne pouvait leur ravir. »Cf. Ballard (J.), Cahiers du Sud, n³23, 1954.

15. « Nous partîmes le 4 mars (1909) en Égypte pour, en conformité de la décision prise par l'Assemblée provinciale de notre diocèse (...) y recueillir une partie des fonds nécessaires à la fondation d'une école d'agriculture à Adana. Pendant que nous poursuivions en Égypte la réalisation de notre but en faisant des démarches auprès tant de l'union générale de bienfaisance arménienne présidée par S.E. Boghos Pacha Nubar, que des notables de la colonie arménienne, les événements se précipitaient à Adana. » Cf. Mouchegh (Mgr.), Les vêpres ciliciennes, Impr. Della-Rocca, Alexandrie, 1909.

16. Adjemian (L.), Op. cit.

17. Beinin (J.) et Lockman (Z.), Workers on the Nile. Nationalism, Communism, islam and the Egyptian working class, 1882-1952, Princeton University Press, 1987, p. 36. "The Europeans shared the special rights and privileges enjoyed by most foreigners (but not by Ottoman subjects). (...) Although the foreign workers were relatively few in number, enjoyed a status superior to that of the indigenous workers, and often had little longterm stake in the country, they were for a time to play as important role in the development of trade unionism in Egypt. Their privileges, higher wages, higher rate of literacy, and prior experience of both industrial life and industrial conflict allowed them at the turn of the century to engage in forms of organization and action that were new to Egypt and which indigenous workers were quick to emulate."

18. Documents diplomatiques concernant l'Égypte de Muhammad 'Ali Jusqu'en 1920, Réunis par l'Association égyptienne de Paris, Ed. Ernest Leroux, Paris, 1920. 